\title{
Low-Temperature Charge Transport in Ga-Acceptor Nanowires Implanted by Focused-Ion Beams
}

\author{
S. J. Robinson ${ }^{1,2}$, C. L. Perkins ${ }^{1}$, J. R. Tucker ${ }^{2}$, T. Schenkel ${ }^{3}$, \\ X. W. Wang ${ }^{4}$, T. P. Ma ${ }^{4}$, and T.-C. Shen ${ }^{1}$ \\ ${ }^{1}$ Department of Physics, Utah State University, Logan, UT 84322 \\ ${ }^{2}$ Department of Electrical and Computer Engineering, University of Illinois at Urbana- \\ Champaign, Urbana, IL 61801 \\ ${ }^{3}$ Lawrence Berkeley National Laboratory, Berkeley, CA 94720 \\ ${ }^{4}$ Department of Electrical Engineering, Yale University, New Haven, CT 06520
}

\begin{abstract}
Ga-acceptor nanowires were embedded in crystalline Si using focused-ion beams. The dc current-voltage characteristics of these wires after annealing are highly nonlinear at low temperatures, and a threshold voltage of less than $50 \mathrm{mV}$ is observed independent of $\mathrm{Ga}^{+}$dosage and implant beam overlap. These features suggest a Coulomb blockade transport mechanism presumably caused by a network of Ga precipitates in the substrate. This granular scenario is further supported by measurements of gated nanowires. Nanowires with metallic conductance at low temperatures could be achieved by reducing the current density of the focused-ion beams.
\end{abstract}


Focused-ion beams (FIB) with nanoscale resolution could be a powerful tool to fabricate nanoscale dopant structures in semiconductor substrates. ${ }^{1}$ The fabrication process is considerably simplified since masks are no longer needed, thus the semiconductor surface contamination can be greatly reduced. FIB implantation enables rapid prototyping and structuring of special samples that do not allow application of standard batch fabrication processes. But since the ion current density of the focused-beam systems commercially available is on the order of $1 \mathrm{~A} / \mathrm{cm}^{2}$, about $10^{6}$ times greater than that of a conventional ion implantation, a major concern is the extensive lattice damage caused by ion irradiation at high dose rates and whether post-implant annealing can restore the crystallinity of the silicon substrates without compromising the dimensions of the dopant structures and other pre-fabricated structures. Conventional implantation of $100 \mathrm{keV} \mathrm{Ga}^{+}$ions at a dose of $3 \times 10^{15} \mathrm{~cm}^{-2}$ can achieve more than $80 \%$ dopant activation and a room temperature sheet resistance of $120 \Omega / \mathrm{sq}$ after annealing for $10 \mathrm{~s}$ at $600{ }^{\circ} \mathrm{C}$, while longer times or higher temperatures lead to higher sheet resistances. ${ }^{2}$ Later reports ${ }^{3,4}$ and our measurements (see below) agree with these conclusions. Literature on electrical characterizations of FIB implanted Ga is, however, less consistent. Tamura et al. implanted 300 $\mu \mathrm{m}^{2}$ areas of $\mathrm{Si}(100)$ substrates up to $5 \times 10^{15}$ ions $/ \mathrm{cm}^{2}$ with a $1-\mu \mathrm{m}$ diameter, $50 \mathrm{keV} \mathrm{Ga}{ }^{+}$beam at a current density of $0.1 \mathrm{~A} / \mathrm{cm}^{2}$, yielding $\sim 80 \%$ carrier activation after annealing at $550{ }^{\circ} \mathrm{C}$ for 5 min. ${ }^{5}$ However, Iwano et al. implanted single-pixel lines at an estimated dose of $6 \times 10^{14}$ ions $/ \mathrm{cm}^{2}$ with a $100 \mathrm{keV} \mathrm{Ga}{ }^{+}$beam of $0.1-\mu \mathrm{m}$ beam diameter reported only $5 \%$ activation, after an anneal of $30 \mathrm{~min}$ at $700{ }^{\circ} \mathrm{C} .{ }^{6}$ Furthermore, Iwano et al. concluded that below $50 \mathrm{~K}$ the conductance of the FIB wire can be described by one-dimensional (1D) variable range hopping (VRH). ${ }^{6,7}$ In doped semiconductor systems, VRH has often been used to interpret electron transport on the insulator side of the metal-insulator transition when carrier density is less than a critical value. ${ }^{8}$ It is, therefore, unclear why the FIB defined lines with such high Ga concentrations are not metallic or if it can be metallic with optimized implantation doses, dose rates and post-implantation annealing processes.

In this letter we present our experimental investigation on the conductivity of the Ga FIB wires at low temperatures. We find that the wires exhibit non-metallic transport behavior. The dc currentvoltage $(I-V)$ characteristics are highly nonlinear. A conductance threshold is observed below 2 $\mathrm{K}$. These transport behaviors can be understood in terms of multiple tunnel junctions with Coulomb blockade islands formed from gallium precipitates.

Figure 1(a) shows part of a 2-terminal device contact array consisting of 200 pairs of interdigitated finger contacts. ${ }^{9}$ A conventional implant of $40-50 \mathrm{keV} \mathrm{Ga}{ }^{+}$ions at a dose of $1 \times 10^{15}$ $\mathrm{cm}^{-2}$ was used to define this array in a p-type substrate (B-doped, $3 \times 10^{17} \mathrm{~cm}^{-3}$ ) through an $11-\mathrm{nm}$ oxide layer. ${ }^{10}$ The beam density during contact implantation is $0.3 \mu \mathrm{A} / \mathrm{cm}^{2}$. After a 5 -min anneal at $550{ }^{\circ} \mathrm{C}$, the average parasitic sheet resistance of our device template, based on $800-\mu \mathrm{m}$ long and $1-\mu \mathrm{m}$ wide test lines, is $720 \Omega / \mathrm{sq}$ at $0.3 \mathrm{~K}$. FIB lines are fabricated across a $1 \mu \mathrm{m}$ gap between two adjacent contact fingers, as shown in Fig. 1(a). The resistance of a FIB line is more than two orders of magnitude higher than the contact resistance at low temperatures, and current leakage through the substrate is negligible below $16 \mathrm{~K}$. FIB line data will therefore not require an adjustment for parasitics.

The conventionally implanted test lines have linear $I-V$ characteristics down to at least $0.3 \mathrm{~K}$, and the change of resistance is less than $1 \%$ from 16 to $0.3 \mathrm{~K}$ as shown in Fig. 1(b). The 
magnetoresistance measured at $0.3 \mathrm{~K}$ increases less than $3.5 \%$ from 0 to $7 \mathrm{~T}$. All these results suggest that the conventionally implanted Ga test lines are 3D metallic conductors. An additional 5 -min annealing at $550{ }^{\circ} \mathrm{C}$ induces an extensive resistance spread from $1.57 \mathrm{k} \Omega / \mathrm{sq}$ to $525 \Omega / \mathrm{sq}$. This is consistent with earlier observations of "reverse annealing" - dopant diffusion and precipitation outdo lattice recovery in both conventional ${ }^{4}$ and FIB implanted samples ${ }^{5}$. Our measurements on conventional implants will now provide a baseline of for the FIB line data reported here.

The FIB implantation was conducted by a FEI Strata-235 Dual Beam system at ion energy of 30 $\mathrm{keV}$ followed by rapid thermal annealing at 550 to $700^{\circ} \mathrm{C}$. Although the beam diameter can be as small as $6 \mathrm{~nm}$ by setting the aperture at $1 \mathrm{pA}$, the actual beam size depends upon how well the beam is focused and a precise estimate is very difficult. In this report we focus on the results from FIB currents of $2 \mathrm{pA}$ and $30 \mathrm{pA}$, where ion sputtering and side-wall formation is negligible based on our atomic force microscopy. Assuming a beam diameter of $\sim 10 \mathrm{~nm}$ at $2 \mathrm{pA}$, the current density is $\sim 2.5 \mathrm{~A} / \mathrm{cm}^{2}$, which is more than $10^{7}$ times greater than the current density used in fabricating the contact arrays. Charge transport was conducted through single-pixel lines implanted between two adjacent fingers; but 1- $\mu$ m wide squares was also implanted for comparison. To ensure sufficient overlap in single-pixel-line doping, the step size of the beam was set to be $7.4 \mathrm{~nm}$ and the dwell time at each step was $0.3 \mu \mathrm{s}$. For single-pixel lines to be conductive, we found that the line-dose had to be more than $1 \times 10^{11} \mathrm{ion} / \mathrm{cm}$, or more than $10^{4}$ passes, at a beam current of $2 \mathrm{pA}$. This overwriting spreads the line more than the expected beam-width. The FIB-implanted line can be seen, as shown in Fig. 1(a), from the secondary electron emission images of the oxide layers, and they are typically $100 \mathrm{~nm}$ wide.

$I-V$ characteristics of the FIB lines are highly nonlinear at $0.3 \mathrm{~K}$, even when the estimated $\mathrm{Ga}^{+}$ dose is between $8.6 \times 10^{15}$ to $3.5 \times 10^{17}$ ions $/ \mathrm{cm}^{2}$ which is much greater than the dose of the $\mathrm{Ga}^{+}-$ implanted contacts. In contrast to VRH systems, no ohmic regime was observed at low voltage bias and electric field. ${ }^{11}$ Instead, all of the $I-V$ data in Fig. 2 have a threshold voltage, $V_{\text {th }}$. The conductance drops exponentially below $\mathrm{V}_{\text {th }}$ while increases nonlinearly above $\mathrm{V}_{\text {th }}$. Figure 3 shows the temperature behavior of the differential conductance of a typical FIB wire. At higher temperatures, thermal activation increases and eventually suppresses the threshold, as depicted in the upper inset of Fig.3. A voltage threshold of this kind, and its thermal suppression, is characteristic of Coulomb blockade in charge transport.

Non-linear $I-V$ characteristics with a conduction threshold have been observed in many granular systems including Pt nanowires ${ }^{12}$, polymer nanofibers ${ }^{13}$, nitride films with $\mathrm{Al}$ nanocrystals ${ }^{14}$ and thin films of colloidal $\mathrm{Co}^{15}, \mathrm{Au}{ }^{16,17}$, and $\mathrm{PbSe}^{18}$ nanoparticles. The transport of these systems can be modeled by a disordered network of tunnel junctions. The disorder originates from both the geometry of the conducting islands in the network and the offsets of their Fermi levels due to nearby random charges. Assuming regular metal-dot arrays, Middleton and Wingreen found that random offset charges lead to a conduction threshold which is proportional to the sizes of the arrays. ${ }^{19}$ Beyond threshold, the predicted current follows the relation $I=I_{0}\left(V / V_{t h}-1\right)^{\varsigma}$ where $\zeta$ $=1,5 / 3$ in $1 \mathrm{D}$ and $2 \mathrm{D}$, respectively. ${ }^{19}$ By fitting our data to this expression, we find $15 \mathrm{mV} \leq V_{t h} \leq 50 \mathrm{mV}$ and $1.2 \leq \zeta \leq 2.3$. Note that despite the dosage range from $8.5 \times 10^{15}$ to $3.5 \times 10^{17} \mathrm{ion} / \mathrm{cm}^{2}$, two different FIB currents, and two types of FIB patterns, the dispersion of $V_{t h}$ 
of the seven samples is relatively small and the range of $\zeta$ is similar to those in other granular systems. $^{13,17}$

Lattice damage induced by single-pixel FIB lines have been investigated by Chu et al. ${ }^{20}$ with ion current, dose and current density similar to our work. Their cross-sectional TEM images clearly show that the disordered $\sim 70 \mathrm{~nm}$ cylindrical cross section of the original implanted line becomes a $\sim 25 \mathrm{~nm}$ square after a $60 \mathrm{~s}$ anneal at $550^{\circ} \mathrm{C}$. Regions of very high Ga concentration are formed as the silicon recrystallizes around the highly disordered FIB implanted areas. Even after 15 min annealing at $800{ }^{\circ} \mathrm{C}$, disordered $\mathrm{Ga}$ precipitation regions of various sizes can be observed in plane-view TEM images. ${ }^{5}$ It is plausible that, under the right annealing conditions, the high Ga regions can form a network of Coulomb islands and tunnel junctions. With a sufficient applied voltage, a steady current can be established by holes tunneling from island to island. The low dispersion of $\mathrm{V}_{\text {th }}$ among our samples suggests that geometry disorder does not affect $\mathrm{V}_{\text {th }}$ significantly at low temperatures. ${ }^{21}$ The fact that the threshold voltage is similar in square patterns and multiple-pass-single-pixel lines rules out insufficient overlapping of the ion irradiation to be the cause of non-ohmic behavior. The lower inset of Fig. 3 shows that zero-bias conductivity of four samples follows $\exp \left[-2 \sqrt{U / k_{B} T}\right]$ with similar activation energy U. Such temperature dependence suggests tunneling through non-uniform-sized grains ${ }^{22}$, which would be the case if the grains are formed from Ga precipitation after rapid annealing.

To further examine this granular scenario, 30-nm layers of $\mathrm{Si}_{3} \mathrm{~N}_{4}$ were deposited at room temperature by jet vapor deposition (JVD) ${ }^{23}$ over the 11-nm oxide layer of the Ga-implanted substrates. Gates were made by applying silver paste over the nitride layer to cover the finger contact region containing the FIB line connection. We find that positive gate voltages do not have any effect on the subthreshold current, while a sufficient negative gate voltage can eliminate the voltage threshold and leads to a linear $I-V$ as shown in Fig. 4. We can understand this behavior by assuming that Ga precipitation contains a high density of holes. These holes will screen out any electric field induced by the gate voltage. Hence the common gate cannot modulate the tunneling current. However, a large negative gate bias can accumulate holes in the B-doped substrate allowing classical hole transport between the $\mathrm{p}^{+}$contacts, which overwhelms the tunneling current and results in a linear $I-V$.

In conclusion, we have demonstrated that increasing the FIB implantation dose up to $3.5 \times 10^{17}$ ions $/ \mathrm{cm}^{2}$ leads to nanowires with non-metallic characteristics at low temperatures. Transport signatures point to the presence of Ga precipitates formed in a network of disordered conducting grains. Holes tunneling between grains yields nonlinear $I-V S$ and conductance thresholds. Optimized annealing could reduce Ga precipitation, but diffusion might reduce Ga concentration below the critical value for metallic conductance. A promising solution is to develop FIBs with improved control over the ion-current density in a low density regime ${ }^{24}$, and to adapt scanning probe techniques with dynamic shadow masks for non-invasive imaging and alignment of implant beams to active device regions ${ }^{25}$.

We thank Jeffrey Kline for earlier experimental work and Andrew Minor for technical assistance of the FIB. This work was partially sponsored by the National Science Foundation NIRT Grant No. CCF-0404208. Portions of this work were performed at the Molecular Foundry, Lawrence Berkeley National Laboratory, which is supported by the Office of Science, Office of Basic 
Energy Sciences, U.S. Department of Energy, under Contract No. DE-AC02-05CH11231. FIB work was performed at the National Center for Electron Microscopy (NCEM) at LBNL.

\section{References}

${ }^{1}$ J. Orloff, M. Utlaut and L. Swanson, High Resolution Focused Ion Beams (Kluwer, New York, 2003).

${ }^{2}$ H. B. Harrison, S. S. Iyer, G. A. Sai-Halasz, S. A. Cohen, Appl, Phys. Lett. 51, 992 (1987).

${ }^{3}$ C.-M. Lin, A. J. Steckl, T. P. Chow, Appl. Phys. Lett. 52, 2049 (1988).

${ }^{4}$ S. Gennaro, B. J. Sealy, R. Gwilliam, E. Collart in Proceedings of Seventh International Workshop on: Fabrication, Characterization, and Modeling of Ultra-Shallow Doping Profiles in Semiconductors, Santa Cruz, CA, 4/27-5/1, 2003.

${ }^{5}$ M. Tamura, S. Shukuri, M. Moniwa, Appl. Phys. A 39, 183 (1986).

${ }^{6}$ H. Iwano, S. Zaima, Y. Koide, Y. Yasuda, J. Vac. Sci. Technol. B 11, 61 (1993).

${ }^{7}$ H. Iwano, S. Zaima, Y. Yasuda, J. Vac. Sci. Technol. B 16, 2551 (1998).

${ }^{8}$ B. I. Shklovskii and A. L. Efros, Electronic Properties of Doped Semiconductors (SpringerVerlag, Berlin, 1984).

${ }^{9}$ T.-C. Shen, J. S. Kline, T. Schenkel, S. J. Robinson, J.-Y. Ji, C. Yang, R.-R. Du, J. R. Tucker, J. Vac. Sci. Technol. B 22, 3182 (2004).

${ }^{10} \mathrm{~N}$-type substrates will provide lateral confinement of the holes in the transport measurement of Ga implanted structures. However, since the substrates are frozen out below $16 \mathrm{~K}$, transport through $p$-type substrates is negligible in our experiments.

${ }^{11}$ D. Shahar and Z. Ovadyahu, Phys. Rev. Lett. 64, 2293 (1990).

${ }^{12}$ L. Rotkina, J.-F. Lin and J. P. Bird, Appl. Phys. Lett. 83, 4426 (2003).

${ }^{13}$ A. N. Aleshin, H. J. Lee, S. H. Jhang, H. S. Kim, K. Akagi, and Y. W. Park, Phys. Rev. B 72, 153202 (2005).

${ }^{14}$ Y. Liu, T. P. Chen, H. W. Lau, J. I. Wong, L. Ding, S. Zhang, S. Fung, Appl. Phys. Lett. 89, 123101 (2006).

${ }^{15}$ C. T. Black, C. B. Murray, R. L. Sandstrom, S. Sun, Science 290, 1131 (2000).

${ }^{16}$ R. Parthasarathy, X.-M. Lin, H. M. Jaeger, Phys. Rev. Lett. 87, 186807 (2001).

${ }^{17}$ K. Elteto, X.-M. Lin, and H. M. Jaeger, Phys. Rev. B 71, 205412 (2005).

${ }^{18}$ H. E. Romero and M. Drndic, Phys. Rev. Lett. 95, 156801 (2005).

${ }^{19}$ A. A. Middleton, N. S. Wingreen, Phys. Rev. Lett. 71, 3198 (1993).

${ }^{20}$ C. H. Chu, Y. F. Hsieh, L. R. Harriott, and H. H. Wade, J. Vac. Sci. Technol. B 9, 3451 (1991).

${ }^{21}$ A. S. Cordan, Y. Leroy, A. Goltzené, A. Pépin, C. Vieu, M. Mejias, and H. Launois, J. Appl. Phys. 87, 345 (2000).

${ }^{22}$ P. Sheng, B. Abeles, Y. Arie, Phys. Rev. Lett. 31, 44 (1973).

${ }^{23}$ T.-P. Ma, IEEE Trans. Electron Dev. 45, 680 (1998).

${ }^{24}$ T. Shinada, S. Okamoto, T. Kobayashi, and I. Ohdomari, Nature 437, 1128 (2005).

${ }^{25}$ A. Persaud, S. J. Park, J. A. Liddle, J. Bokor, I. W. Rangelow, and T. Schenkel, Nano Lett. 5, 1087 (2005). 


\section{Figure captions}

1. (a) SEM images of a Ga implanted 2-terminal template. A FIB line is fabricated across two adjacent finger contacts. (b) Temperature dependence of the resistance of a conventionally Ga implanted line of dimensions $1 \mu \mathrm{m} \times 800 \mu \mathrm{m}$.

2. $I-V$ characteristics at $0.3 \mathrm{~K}$ of (a) single-pixel lines implanted by FIB at $2 \mathrm{pA}$, (b) $30 \mathrm{pA}$ and (c) a 1- $\mu \mathrm{m}$ square pattern implanted by FIB at $30 \mathrm{pA}$. Threshold voltage for (a), (b) and (c) is 47 , 50 and $20 \mathrm{mV}$, respectively.

3. Temperature dependence of the differential conductance of the sample presented in Fig. 2(c). From top to bottom, $\mathrm{T}=4.07,1.60,1.17$, and $0.293 \mathrm{~K}$. The upper inset shows temperature dependence of the threshold voltage of this sample. The lower inset shows zero-bias conductance as a function of $1 / \sqrt{T}$ of 4 samples.

4. Negative gate effects on the $I-V$ characteristics of a gated FIB line at $0.3 \mathrm{~K}$. Positive gate voltages have no effect on $I_{d}$ in the $V_{d}$ range presented here. 


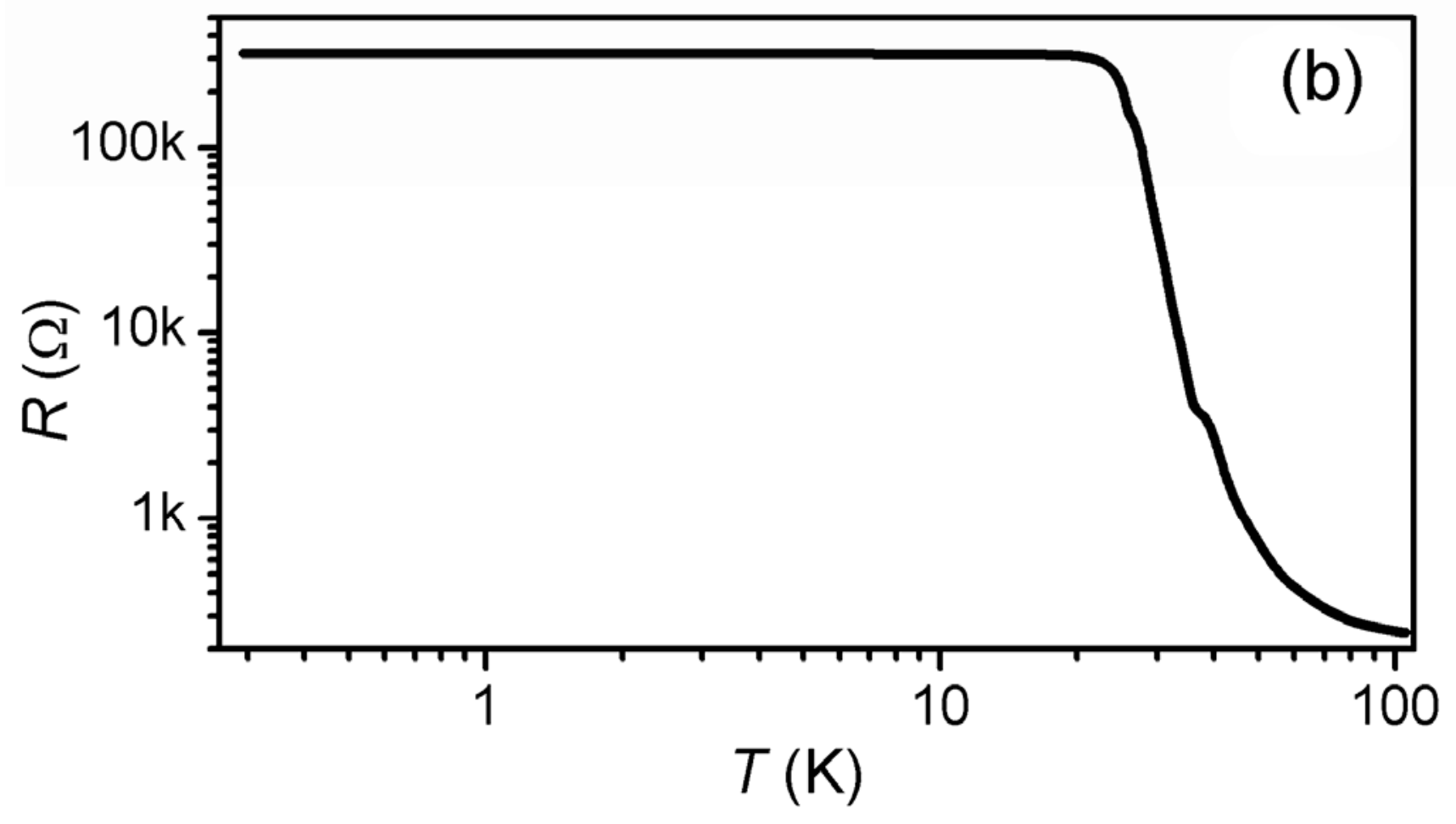




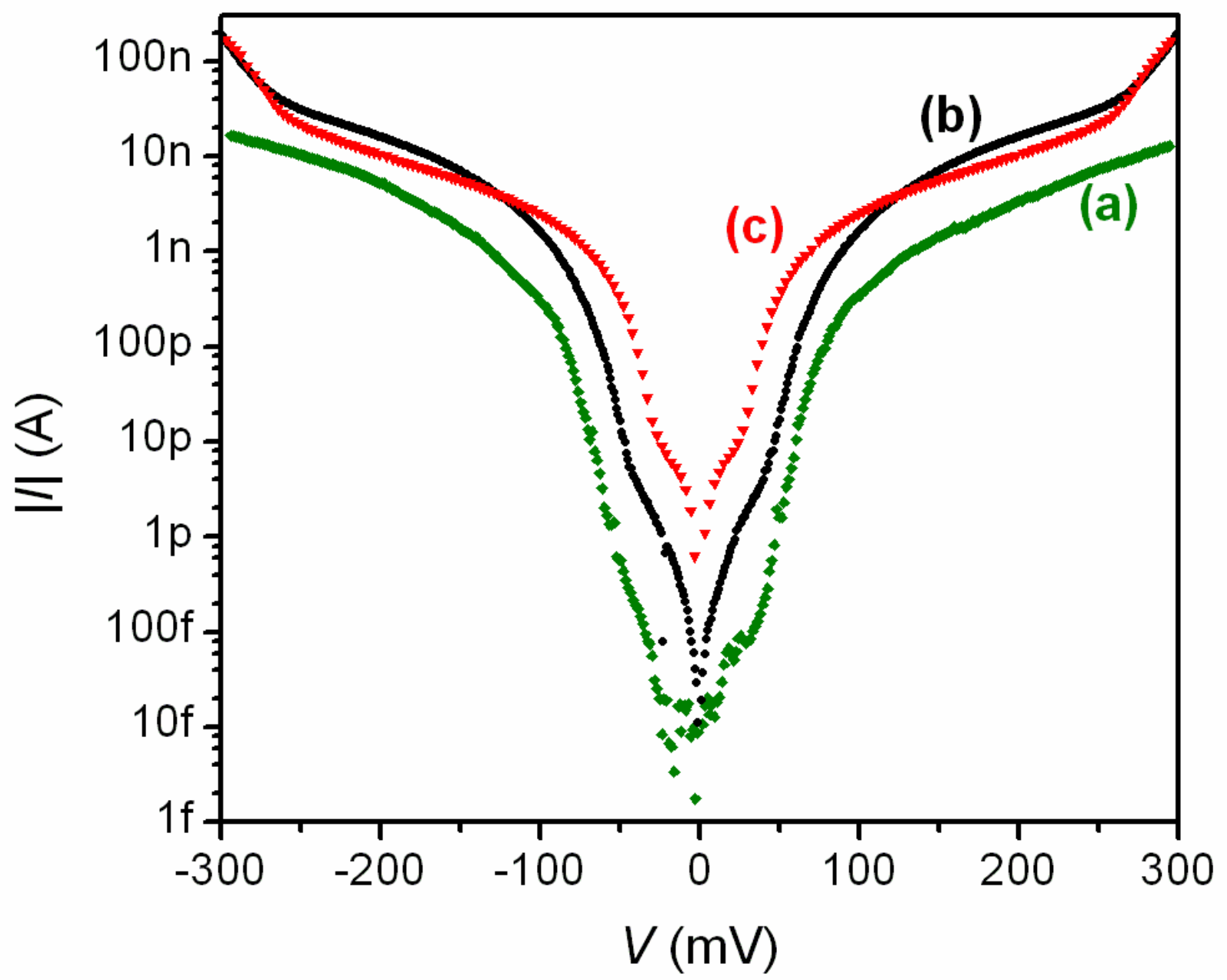

Figure 2 


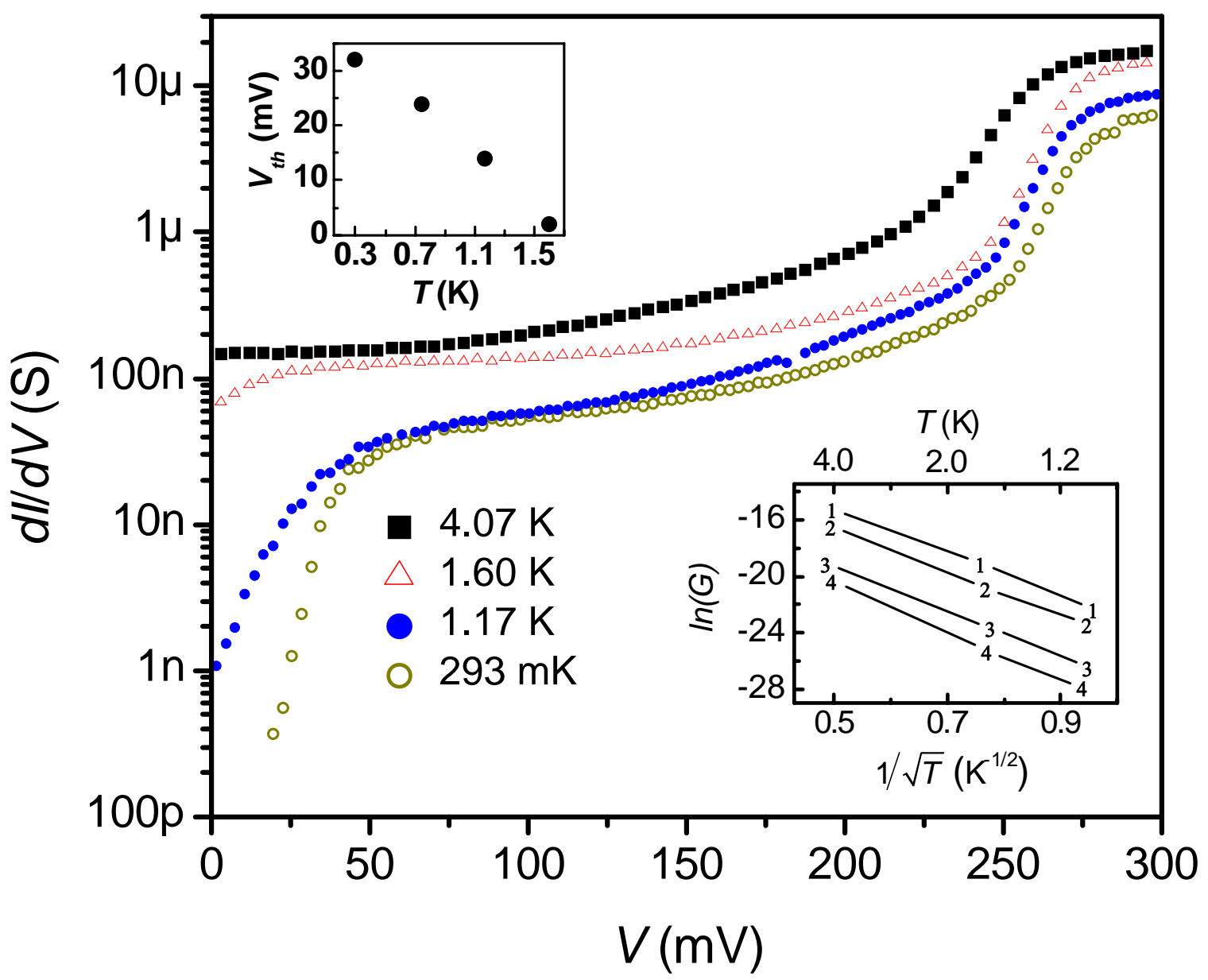

Figure 3 


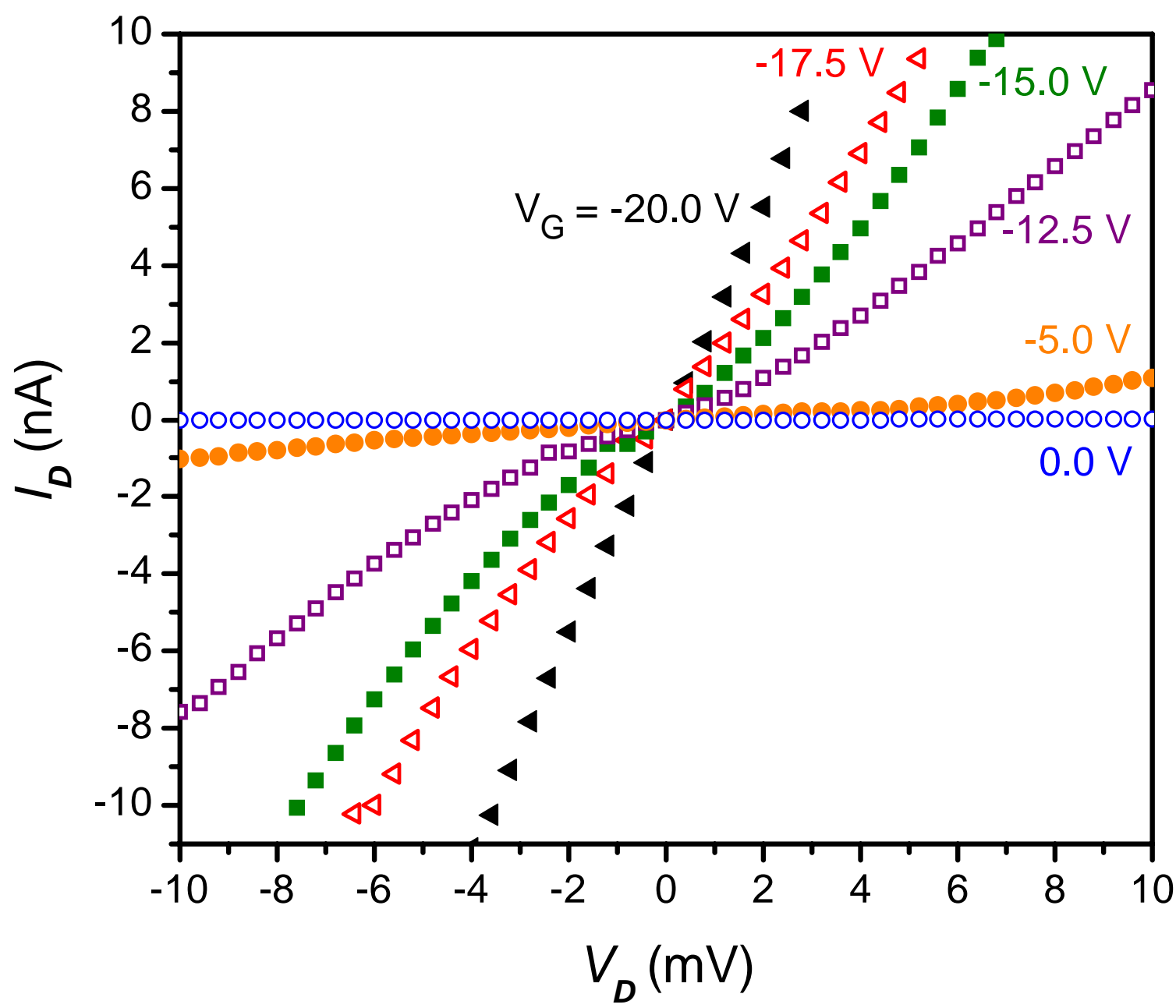

Figure 4 